\title{
Nucleon resonance studies in phenomenological analysis of the CLAS data on double charged pion photo and electroproduction.
}

\author{
V.I. Mokeev ${ }^{*, \dagger}$ and V.D. Burkert* \\ ${ }^{*}$ Thomas Jefferson National Accelerator Facility, Newport News, Virginia 23606, USA \\ ${ }^{\dagger}$ Skobeltsyn Nuclear Physics Institute at Moscow State University, 119899 Moscow, Vorobevy gory,
} Russia

\begin{abstract}
First comprehensive data on the evolution of nucleon resonance photocouplings with photon virtuality $Q^{2}$ are presented for excited proton states in the mass range from 1.4 to $2.0 \mathrm{GeV}$.
\end{abstract}

Keywords: nucleon resonances, meson photo- and electro production

PACS: PACS number(s): 13.40.Gp, 13.60.Le, 14.20.Gk

\section{INTRODUCTION.}

Comprehensive studies of nucleon resonances were carried out in analysis of CLAS data on double charged pion production by real and virtual photons $[3,4,5]$. The analysis was performed within the framework of the 2005 version of JLAB-MSU model in the following referred to as JM05 [1].

\section{JM05 PHENOMENOLOGICAL MODEL.}

We have developed a phenomenological model, that incorporates particular mesonbaryon mechanisms based on their manifestations in observables: as enhancements in invariant mass distributions, sharp forward/backward slopes in angular distributions. Our model is currently limited to the $\mathrm{p} \pi^{-} \pi^{+}$final state and incorporates particular meson-baryon mechanisms needed to describe $\pi^{+} \mathrm{p}, \pi^{+} \pi^{-}, \pi^{-} \mathrm{p}$ invariant masses and $\pi^{-}$angular distribution. These cross sections $[3,4,5]$ were analyzed in the hadronic mass range from 1.41 to $1.89 \mathrm{GeV}$ and in four $Q^{2}$ bins centered at $0 ., 0.65,0.95,1.30 \mathrm{GeV}^{2}$. Double charged pion production was described by the superposition of quasi-two-body channels:

$$
\begin{aligned}
& \gamma p \rightarrow \pi^{-} \Delta^{++} \rightarrow \pi^{-} \pi^{+} p, \\
& \gamma p \rightarrow \pi^{+} \Delta^{0} \rightarrow \pi^{+} \pi^{-} p, \\
& \gamma p \rightarrow \rho^{0} p \rightarrow \pi^{+} \pi^{-} p \\
& \gamma p \rightarrow \pi^{+} D_{13}^{0}(1520) \rightarrow \pi^{+} \pi^{-} p . \\
& \gamma p \rightarrow \pi^{+} F_{15}^{0}(1685) \rightarrow \pi^{+} \pi^{-} p, \\
& \gamma p \rightarrow \pi^{-} P_{33}^{++}(1600) \rightarrow \pi^{-} \pi^{+} p .
\end{aligned}
$$


The production amplitudes for the first three quasi-two-body mechanisms (1-3) were treated as sums of $N^{*}$ excitations in the $s$-channel and non-resonant mechanisms described in Ref [2]. The quasi-two-body mechanisms (4-6) were entirely non-resonant $[1,7]$. In reactions (1-3) all well established 4 star resonances with observed decays to the two pion final states were included as well as the 3 -star states $D_{13}(1700), P_{11}(1710)$, $P_{33}(1600)$, and $P_{33}(1920)$. $N^{*}$ electromagnetic transition form factors were fitted to the data. Hadronic couplings for $N^{*} \rightarrow \pi \Delta$ and $\rho p$ decays were taken from the analyses of experiments with hadronic probes, except for $P_{33}(1600), P_{13}(1720)$, the candidate $3 / 2^{+}(1720), D_{13}(1700), P_{33}(1920), F_{35}(1905)$, and $F_{37}(1950)$ states. Poorly known hadronic decay parameters for these states were fitted to the data.

Remaining residual mechanisms were parametrized as the set of exchange terms. Parametrization of these exchange amplitudes is described in [1, 6].

In the JM05 model we succeeded in describing all available CLAS data on unpolarized observables in $2 \pi$ photo and electroproduction. These results are presented in [7]. We found no need for remaining mechanisms of unknown dynamics. Therefore, the quality of the CLAS data allow us to establish all significant mechanisms in $2 \pi$ production, implementing particular meson-baryon diagrams as determined from the data fit.

\section{$N^{*}$ ANALYSIS IN $2 \pi$ PHOTO AND ELECTROPRODUCTION.}

We fit all available CLAS data on $2 \pi$ photo and electroproduction at $\mathrm{W} \leq .1 .9 \mathrm{GeV}$ and $Q^{2}$ from 0 to $1.5 \mathrm{GeV}^{2}$ within the framework of JM05. $N^{*}$ photocouplings were sampled according to the normal distribution around the values, obtained in JM03 version $[1,2]$. Poorly known masses and hadronic couplings were also fluctuated inside the uncertainties established in experiments with hadronic probes. For each trial set of model parameters we calculated all kind of single differential cross sections in all available $\mathrm{W}$ and $Q^{2}$ bins. From comparison between calculated and measured single differential cross sections $\chi^{2} /$ d.p. were estimated. We isolated a bunch of calculated cross sections inside the data uncertainties, applying restriction $\chi^{2} \leq \chi_{t h}^{2}$, where $\chi_{t h}^{2}$ is a predetermined maximal allowed value. A reasonable description of all cross sections was achieved.

$N^{*}$ photocouplings for selected cross sections were averaged and mean values were treated as extracted from the data fit, while dispersions were assigned to photocoupling uncertainties. In this way we obtained the photocouplings for the states: $P_{11}(1440)$, $D_{13}(1520), S_{31}(1620), S_{11}(1650), P_{33}(1600), F_{15}(1680), D_{13}(1700), D_{33}(1700)$, candidate $3 / 2^{+}(1720), P_{13}(1720), F_{35}(1905), P_{33}(1920)$, and $F_{37}(1950)$ [7]. In Fig. 1 we present the photocouplings for the well studied $D_{13}(1520)$ state in comparison with previously available data.

For the first time, we determine the electrocouplings for high lying $N^{*}$, which preferably decay with $2 \pi$ emission: $D_{13}(1700), D_{33}(1700)$, candidate $3 / 2^{+}(1720), P_{13}(1720)$, $F_{35}(1905), P_{33}(1920)$, and $F_{37}(1950)$. In Fig. 2 we present photo- and electrocouplings for the $D_{33}(1700)$ and $P_{13}(1720)$ states extracted from the CLAS $2 \pi$ data, as well as couplings obtained in previous studies of $1 \pi$ production. 


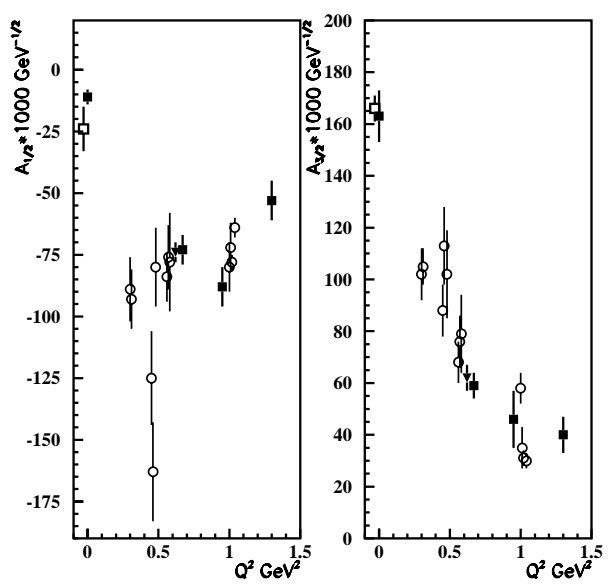

FIGURE 1. $D_{13}(1520)$ photocouplings extracted from the analysis of CLAS $2 \pi$ data [3-5] (filled squares) in comparison with world data [1] (open circles) and results of analysis of the CLAS $1 \pi$ and $2 \pi$ data combined [6] (filled triangles).
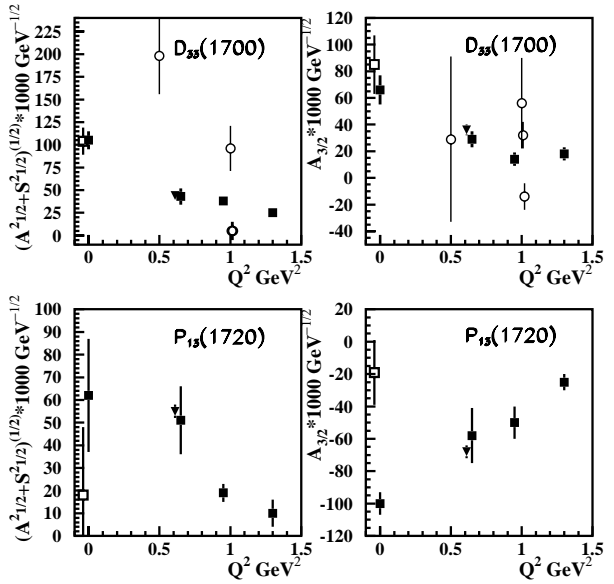

FIGURE 2. $D_{33}(1700), P_{13}(1720)$ photocouplings extracted from analysis of CLAS $2 \pi$ data in comparison with previous results. Symbols are the same as in the Fig. 1.

\section{REFERENCES}

1. V. Mokeev et. al.,hep - ph/0512164, JLAB-PHY-06-2 be published in Proc of NSTAR2005 Workshop.

2. V. D Burkert, et. al., Phys. of Atom. Nucl., 66, 2199-2208 (2003).

3. M. Ripani et. al., Phys. Rev. Lett., 91, 022002-1-022002-6 (2003).

4. M.Bellis, et.al., Proceedings of NSTAR2004 workshop, March 24-27, 2004, Grenoble, France, World Scientific, Grenoble, 2004, pp 139-149.

5. CLAS Physics Data Base, http : //clasdb3.jlab.org.

6. I. G. Aznauryan et. al., Phys. Rev., C72, 045201-1-045201-9 (2005).

7. V.I.Mokeev http://hadron.physics.fsu.edu/nstar/scientificProg.htm 